\title{
Growth of the solutions of some $q$-difference differential equations
}

Hong-Yan $\mathrm{Xu}^{1{ }^{*}}$, Lian-Zhong Yang ${ }^{2}$ and Hua Wang ${ }^{1}$

\section{"Correspondence:} xhyhhh@126.com

1 Department of Informatics and Engineering, Jingdezhen Ceramic Institute, Jingdezhen, Jiangxi 333403, China

Full list of author information is available at the end of the article

\begin{abstract}
In view of Nevanlinna theory, we study the growth and poles of solutions of some complex q-difference differential equations. We obtain the estimates on the Nevalinna order, the lower order, and the counting function of poles for meromorphic solutions of such equations.
\end{abstract}

MSC: 39A13; 30D35

Keywords: growth; $q$-difference differential equation; pole

\section{Introduction and main results}

In this paper, the fundamental theorems and the standard notations of the Nevanlinna value distribution theory of meromorphic functions will be used (see Hayman [1], Yang [2] and Yi and Yang [3]). For a meromorphic function $f(z)$, we also use $S(r, f)$ to denote any quantity satisfying $S(r, f)=o(T(r, f))$ for all $r$ outside a possible exceptional set $E$ of finite logarithmic measure $\lim _{r \rightarrow \infty} \int_{[1, r) \cap E} \frac{d t}{t}<\infty$, and a meromorphic function $a(z)$ is called a small function with respect to $f$, if $T(r, a)=S(r, f)=o(T(r, f))$.

In 1925, Ritt [4] gave the form of solutions of the Schrödinger equation

$$
f(c z)=R(f(z))
$$

where $c \in \mathbb{C}, c \neq 0,1$, and $R(f)$ is a rational function in $f$. In 1983, Rubel [5] posed the following question:

What can be said about the more general equation

$$
f(c z)=R(z, f(z)),
$$

where $R(z, f)$ is rational in both variables?

Later, Ishizaki [6] and Wittich [7] investigated the existence of meromorphic solutions of the equation of the following form:

$$
f(c z)=a(z) f(z)+b(z)
$$

where $a(z)$ and $b(z)$ are meromorphic functions.

In 2002, Gundersen et al. [8] studied the growth of meromorphic solutions of $q$ difference equations and obtained results as follows.

(c) $2015 \mathrm{Xu}$ et al. This article is distributed under the terms of the Creative Commons Attribution 4.0 International License (http://creativecommons.org/licenses/by/4.0/), which permits unrestricted use, distribution, and reproduction in any medium, provided you give appropriate credit to the original author(s) and the source, provide a link to the Creative Commons license, and indicate if changes were made. 
Theorem 1.1 ([8], Theorem 3.2) Suppose that $f$ is a transcendental meromorphic solution of an equation of the form

$$
f(c z)=R(z, f(z))=\frac{\sum_{j=0}^{p} a_{j}(z) f(z)^{j}}{\sum_{j=0}^{q} b_{j}(z) f(z)^{j}}
$$

with meromorphic coefficients $a_{j}(z), b_{j}(z)$ are of growth $S(r, f)$, and a constant $c(|c|>1)$, assuming that $d:=\max \{p, q\} \geq 1, a_{p}(z) \neq 0, b_{q}(z) \neq 0$, and that $R(z, f(z))$ is irreducible in $f$. Then $\rho(f)=\frac{\log d}{\log |c|}$, where $\rho(f)=\lim \sup _{r \rightarrow+\infty} \frac{\log \log T(r, f)}{\log r}$.

Theorem 1.2 ([8], Theorem 3.4) Let c be a complex constant satisfying $|c|>1$, and suppose that $f$ is a nonconstant meromorphic solution of a functional equation of the form

$$
A(c z, f(c z))=B(z, f(z)),
$$

where $A(z, y)$ and $B(z, y)$ are rational functions with meromorphic coefficients of growth $S(r, f)$ such that $A(z, y)$ and $B(z, y)$ are irreducible in $y$. If $0<a:=\operatorname{deg}_{f} A \leq \operatorname{deg}_{f} B=: b$, then $\rho(f)=\frac{\log b-\log a}{\log |c|}$.

In 2012, Beardon [9] studied entire solutions of the generalized function equation

$$
f(q z)=q f(z) f^{\prime}(z), \quad f(0)=0,
$$

where $q$ is a non-zero complex number. To state the results of Beardon [9], we first introduce some notations as follows.

Let the formal series $\mathcal{O}$ and $\mathcal{I}$ be defined by

$$
\mathcal{O}:=0+0 z+0 z^{2}+\cdots, \quad \mathcal{I}:=0+1 z+0 z^{2}+0 z^{3}+\cdots,
$$

and the sets $\mathcal{K}_{p}=\left\{z: z^{p}=p+2\right\}(p=1,2, \ldots)$, and $\mathcal{K}=\mathcal{K}_{1} \cup \mathcal{K}_{2} \cup \cdots$. Thus, we see that $\mathcal{K}_{p}$ contains $p$ elements and $|z|=r_{p}$, for $z \in \mathcal{K}_{p}$, where $r_{p}=(p+2)^{\frac{1}{p}}$. Since $p \in \mathbb{N}_{+}$, we have $|z|>1$. Since $\frac{\log (x+2)}{x}$ is decreasing as $x>1$, we have $r_{1}>r_{2}>\cdots>1$, and $r_{p} \rightarrow 1$ as $p \rightarrow \infty$. Based on the above notations, Beardon obtained two main theorems as follows.

Theorem 1.3 ([9]) Any transcendental solution $f$ of $(1)$ is of the form

$$
f(z)=z+z\left(b z^{p}+\cdots\right)
$$

where $p$ is a positive integer, $b \neq 0$ and $q \in \mathcal{K}_{p}$. In particular, if $q \notin \mathcal{K}$, then the only formal solutions of (1) are $\mathcal{O}$ and $\mathcal{I}$.

Theorem 1.4 ([9]) For each positive integer $p$, there is a unique real entire function

$$
F_{p}(z)=z\left(1+z^{p}+b_{2} z^{2 p}+b_{3} z^{3 p}+\cdots\right)
$$

which is a solution of (1) for each $q \in \mathcal{K}_{p}$. Further, if $q \in \mathcal{K}_{p}$, then the only transcendental solutions of $(1)$ are the linear conjugates of $F_{p}$. 
Recently, Zhang [10] further studied the growth of solutions of (1) and obtained the following theorem.

Theorem 1.5 ([10], Theorem 1.1) Suppose that $f$ is a transcendental solution of (1) for $q \in \mathcal{K}$, then we have

$$
\rho(f) \leq \frac{\log 2}{\log |q|}
$$

where

$$
\rho(f)=\limsup _{r \rightarrow \infty} \frac{\log T(r, f)}{\log r} .
$$

Regarding Theorem 1.5, Zhang [10] asked the following question: Is the order of transcendental solutions of (1) exactly $\rho(f) \leq \frac{\log 2}{\log |q|}$ ?

In this paper, we further investigate the growth of solution of some class of $q$-difference differential equation and obtain the following results.

Theorem 1.6 Suppose that $f$ is a solution of equation

$$
f(q z)^{n}=q f(z)\left[f^{(j)}(z)\right]^{s}
$$

where $q \in \mathcal{K}$ and $n, s, j \in N_{+}$. Iff is a transcendental entire function, then $n \leq s+1$ and the order off satisfies

$$
\rho(f) \leq \frac{\log (s+1)-\log n}{\log |q|} .
$$

The following example shows that (2) has non-transcendental entire function solution.

Example 1.1 Let $q=2, n=2, j=1$, and $s=2$, then $f(z)=2 z^{2}$ satisfies equation

$$
f(2 z)^{2}=2 f(z)\left(f^{\prime}(z)\right)^{2} .
$$

The following example shows that (2) also has a transcendental entire function solution.

Example 1.2 Let $q=3, n=2, j=1$, and $s=5$, then $f(z)=\exp \left\{3^{-\frac{1}{5}} z\right\}$ satisfies the equation

$$
f(3 z)^{2}=3 f(z)\left(f^{\prime}(z)\right)^{5},
$$

and

$$
\rho(f)=1=\frac{\log 6-\log 2}{\log 3} .
$$

Remark 1.1 Thus, a question arises naturally: Does (2) have a transcendental meromorphic solution?

When the constant $q$ of the right of (2) is replaced by a function, the following example shows that the equation has a transcendental meromorphic solution. 
Example 1.3 Let $f(z)=\frac{e^{z}}{z^{2}}$ and $q=2$, then $f(z)$ satisfies the equation

$$
f(2 z)=\frac{z^{3}}{4 z-8} f(z) f^{\prime}(z)
$$

and the order is

$$
\frac{\log 2-\log 1}{\log |2|}=\rho(f)=1 \leq \frac{\log 3-\log 1}{\log 2} .
$$

Thus, we have the following theorems.

Theorem 1.7 Let $f$ be a transcendental solution of the equation

$$
f(q z)^{n}=\varphi_{1}(z) f(z)\left[f^{(j)}(z)\right]^{s}
$$

where $q$ is a non-zero complex number and $|q|>1, n, j$, s are positive integers and $\varphi_{1}(z)$ is a rational function. Iff is an entire function, then $n \leq s+1$ and

$$
\rho(f) \leq \frac{\log (s+1)-\log n}{\log |q|} .
$$

Furthermore, if $n=1$ and $f$ is a meromorphic function with infinitely many poles, then we have

$$
\frac{\log (s+1)}{\log |q|} \leq \rho(f) \leq \frac{\log (s j+s+1)}{\log |q|} .
$$

Theorem 1.8 Let $f$ be a transcendental solution of the equation

$$
f(q z)^{n}=\varphi_{2}(z) f(z)\left[f^{(j)}(z)\right]^{s}
$$

where $q$ is a complex number and $|q|>1, n, j$, s are positive integers and $\varphi_{2}(z)$ is a small function with respect to $f$. Iff is a meromorphic function with $\bar{N}(r, f)=S(r, f)$, then $n<s+1$ and $f$ satisfies

$$
\rho(f) \leq \frac{\log (s+1)-\log n}{\log |q|} .
$$

Furthermore, if $n=1$ and $f$ has infinitely many poles with $\bar{N}(r, f)=S(r, f)$, and the number of distinct common poles off and $\frac{1}{\varphi_{2}}$ is finite, then we have

$$
\rho(f)=\frac{\log (s+1)}{\log |q|}
$$

The following example shows that (4) has a transcendental meromorphic solution $f$ with the order $\rho(f)=\frac{\log (s+1)}{\log |q|}$.

Example 1.4 Let $n=j=s=1$ and $q=2$, then $f(z)=\frac{(z-1) e^{z}}{z}$ satisfies the equation

$$
f(2 z)=\frac{2 z^{2}-z}{2 z-1} f(z) f^{\prime}(z)
$$


where $\varphi_{2}(z)=\frac{2 z^{2}-z}{2 z-1}$ with $T\left(r, \varphi_{2}\right)=S(r, f)$ and the order of $f(z)$ satisfies

$$
\rho(f)=1=\frac{\log 2-\log 1}{\log 2} .
$$

Let $p(z)=p_{k} z^{k}+p_{k-1} z^{k-1}+\cdots+p_{1} z+p_{0}$, where $p_{k}(\not \equiv 0), \ldots, p_{0}$ are complex constants. Now, we investigate the growth of solutions of such equations, where $q z$ is replaced by $p(z)$ in (2)-(4), and we obtain the following result.

Theorem 1.9 Let $f$ be a transcendental solution of equation

$$
f(p(z))^{n}=\varphi_{3}(z) f(z)\left[f^{(j)}(z)\right]^{s}
$$

where $k \geq 2, n, j$, s are positive integers and $\varphi_{3}(z)$ is a small function with respect to $f$. If $f$ is a transcendental meromorphic function and $n<s j+s+1$, then $f$ satisfies

$$
T(r, f)=O\left((\log r)^{\alpha}\right), \quad \alpha=\frac{\log (s j+s+1)-\log n}{\log k} .
$$

Recently, there were many results on meromorphic solutions of complex functional equations (see [11-20]). In 2007, Barnett et al. [21] firstly established an analog of the logarithmic derivative lemma on $q$-difference operators. In 2010, by applying their theorems, Zheng and Chen [22] considered the growth of meromorphic solutions of $q$-difference equations and obtained results which extended some theorems given by Heittokangas et al. [23].

Theorem 1.10 ([22], Theorem 2) Suppose that $f$ is a transcendental meromorphic solution of equation

$$
\sum_{j=1}^{n} a_{j}(z) f\left(q^{j} z\right)=R(z, f(z))=\frac{P(z, f(z))}{Q(z, f(z))},
$$

where $q \in \mathbb{C},|q|>1$, the coefficients $a_{j}(z)$ are rational functions and $P, Q$ are relatively prime polynomials in $f$ over the field of rational functions satisfying $p=\operatorname{deg}_{f} P, t=\operatorname{deg}_{f} Q$, $d=p-t \geq 2$. Iff has infinitely many poles, then for sufficiently large $r, n(r, f) \geq K d^{\frac{\log r}{\log |q|}}$ holds for some constant $K>0$. Thus, the lower order of $f$, which has infinitely many poles, satisfies $\mu(f) \geq \frac{\log d}{n \log |q|}$, where $\mu(f)=\liminf _{r \rightarrow+\infty} \frac{\log \log T\left(r_{r} f\right)}{\log r}$.

From Theorem 1.10, we further study the growth of the solutions of a class of $q$ difference differential equation and obtain a result as follows.

Theorem 1.11 Suppose that $f$ is a transcendental meromorphic solution of the equation

$$
f(q z) f^{\prime}(z)=R(z, f(z))=\frac{P(z, f(z))}{Q(z, f(z))},
$$

where $q \in \mathbb{C},|q|>1$, and $P, Q$ are relatively prime polynomials in $f$ over the field of rational functions satisfying $p=\operatorname{deg}_{f} P, t=\operatorname{deg}_{f} Q, d=p-t \geq 4$, where the coefficients of $P, Q$ are 
rational functions in $z$. If $f$ has infinitely many poles, then for sufficiently large $r, n(r, f) \geq$ $K(d-1)^{\frac{\log r}{\log |q|}}$ holds for some constant $K>0$. Thus, the lower order of $f$, which has infinitely many poles, satisfies $\mu(f) \geq \frac{\log (d-1)}{\log |q|}$.

Remark 1.2 Under the conditions of Theorem 1.11, by using the same argument as in Theorem 1.8 , we can see that the lower order, the order of $f$, which has infinitely many poles, satisfies

$$
\frac{\log (d-1)}{\log |q|} \leq \mu(f) \leq \rho(f) \leq \frac{\log (d+2)}{\log |q|} .
$$

The following example shows that (6) has a non-transcendental solution.

Example 1.5 Let $q=2$ and $d=3$, then $f(z)=\frac{1}{z^{2}}$ satisfies the equation

$$
f(2 z) f^{\prime}(z)=-\frac{1}{2} z f(z)^{3} .
$$

The following examples show that (6) has transcendental entire and meromorphic solutions.

Example 1.6 Let $q=2$ and $d=3$, then $f(z)=\sin z$ satisfies the equation

$$
f(2 z) f^{\prime}(z)=2 f(z)-2 f(z)^{3} .
$$

Then we have $\mu(f)=\rho(f)=1=\frac{\log (3-1)}{\log 2}$.

Example 1.7 Let $q=2$ and $d=5$, then $f(z)=\frac{1}{z} e^{z^{2}}$ satisfies the equation

$$
f(2 z) f^{\prime}(z)=z^{2}\left(z^{2}-\frac{1}{2}\right) f(z)^{5} .
$$

Then we see that $f$ has finitely many poles and $\mu(f)=\rho(f)=2=\frac{\log (5-1)}{\log 2}$.

Example 1.8 Let $q=2$ and $d=3$, then $f(z)=\frac{1}{\sin z}$ satisfies the equation

$$
f(2 z) f^{\prime}(z)=-\frac{1}{2} f(z)^{3} .
$$

So, $f(z)$ has infinitely many poles and $\mu(f)=\rho(f)=1=\frac{\log (3-1)}{\log 2}$.

Remark 1.3 By comparing Example 1.8 and Theorem 1.11, we pose a question as follows: Whether the condition ' $d=p-t \geq 4$ ' may be relaxed to ' $d \geq 3$ or $d \geq 2$ ' in Theorem 1.11?

\section{Some lemmas}

Lemma 2.1 (Valiron-Mohon'ko [24]) Let $f(z)$ be a meromorphic function. Then for all irreducible rational functions in $f$,

$$
R(z, f(z))=\frac{\sum_{i=0}^{m} a_{i}(z) f(z)^{i}}{\sum_{j=0}^{n} b_{j}(z) f(z)^{j}}
$$


with meromorphic coefficients $a_{i}(z), b_{j}(z)$, the characteristic function of $R(z, f(z))$ satisfies

$$
T(r, R(z, f(z)))=d T(r, f)+O(\Psi(r))
$$

where $d=\max \{m, n\}$ and $\Psi(r)=\max _{i, j}\left\{T\left(r, a_{i}\right), T\left(r, b_{j}\right)\right\}$.

Lemma 2.2 ([3], p.37 or [2]) Let $f(z)$ be a nonconstant meromorphic function in the complex plane and $l$ be a positive integer. Then

$$
N\left(r, f^{(l)}\right)=N(r, f)+l \bar{N}(r, f), \quad T\left(r, f^{(l)}\right) \leq T(r, f)+l \bar{N}(r, f)+S(r, f) .
$$

Lemma 2.3 ([8]) Let $\Phi:(1,+\infty) \rightarrow(0,+\infty)$ be a monotone increasing function, and let $f$ be a nonconstant meromorphic function. If for some real constant $\alpha \in(0,1)$, there exist real constants $K_{1}>0$ and $K_{2} \geq 1$ such that

$$
T(r, f) \leq K_{1} \Phi(\alpha r)+K_{2} T(\alpha r, f)+S(\alpha r, f)
$$

then the order of growth off satisfies

$$
\rho(f) \leq \frac{\log K_{2}}{-\log \alpha}+\limsup _{r \rightarrow \infty} \frac{\log \Phi(r)}{\log r} .
$$

Lemma 2.4 ([25]) Let $f(z)$ be a transcendental meromorphic function and $p(z)=p_{k} z^{k}+$ $p_{k-1} z^{k-1}+\cdots+p_{1} z+p_{0}$ be a complex polynomial of degree $k>0$. For given $0<\delta<\left|p_{k}\right|$, let $\lambda=\left|p_{k}\right|+\delta, \mu=\left|p_{k}\right|-\delta$, then for given $\varepsilon>0$ and for $r$ large enough,

$$
(1-\varepsilon) T\left(\mu r^{k}, f\right) \leq T(r, f \circ p) \leq(1+\varepsilon) T\left(\lambda r^{k}, f\right) .
$$

Lemma $2.5([26,27]$ or $[28])$ Let $g:(0,+\infty) \rightarrow R, h:(0,+\infty) \rightarrow R$ be monotone increasing functions such that $g(r) \leq h(r)$ outside of an exceptional set $E$ with finite linear measure, or $g(r) \leq h(r), r \notin H \cup(0,1]$, where $H \subset(1,+\infty)$ is a set of finite logarithmic measure. Then, for any $\alpha>1$, there exists $r_{0}$ such that $g(r) \leq h(\alpha r)$ for all $r \geq r_{0}$.

Lemma 2.6 ([29]) Let $\psi(r)$ be a function of $r\left(r \geq r_{0}\right)$, positive and bounded in every finite interval.

(i) Suppose that $\psi\left(\mu r^{m}\right) \leq A \psi(r)+B\left(r \geq r_{0}\right)$, where $\mu(\mu>0), m(m>1), A(A \geq 1), B$ are constants. Then $\psi(r)=O\left((\log r)^{\alpha}\right)$ with $\alpha=\frac{\log A}{\log m}$, unless $A=1$ and $B>0$; and if $A=1$ and $B>0$, then for any $\varepsilon>0, \psi(r)=O\left((\log r)^{\varepsilon}\right)$.

(ii) Suppose that (with the notation of (i)) $\psi\left(\mu r^{m}\right) \geq A \psi(r)\left(r \geq r_{0}\right)$. Then for all sufficiently large values of $r, \psi(r) \geq K(\log r)^{\alpha}$ with $\alpha=\frac{\log A}{\log m}$, for some positive constant $K$.

Lemma 2.7 (see [12])

$$
T(r, f(q z))=T(|q| r, f)+O(1)
$$

holds for any meromorphic function $f$ and any non-zero constant $q$. 


\section{Proofs of Theorems 1.6-1.8}

\subsection{The proof of Theorem 1.6}

By Lemma 2.1 and Lemma 2.7, it follows from (2) that

$$
T(|q| r, f(z)) \leq \frac{1}{n} T(r, f)+\frac{s}{n} T\left(r, f^{(j)}(z)\right)+O(1) .
$$

If $f$ is a transcendental entire function, then we have by Lemma 2.2

$$
T(|q| r, f(z)) \leq \frac{1+s}{n} T(r, f)+S(r, f) .
$$

Since $|q|>1$ and $f$ is transcendental, it follows from (8) that $n \leq s+1$. Set $\alpha=\frac{1}{|q|}$, it follows

$$
T(r, f(z)) \leq \frac{1+s}{n} T(\alpha r, f)+S(\alpha r, f) .
$$

By Lemma 2.3, we have $\rho(f) \leq \frac{\log (s+1)-\log n}{\log |q|}$.

\subsection{The proof of Theorem 1.7}

Since $\varphi_{1}(z)$ is a rational function, we have $T\left(r, \varphi_{1}(z)\right)=O(\log r)$. If $f$ is a transcendental entire function, similar to the argument as in Theorem 1.6, we easily get $\rho(f) \leq \frac{\log (s+1)-\log n}{\log |q|}$.

If $f$ is a meromorphic function, by Lemma 2.1, Lemma 2.2, and Lemma 2.7, it follows from (3) that

$$
T(|q| r, f(z)) \leq \frac{s j+s+1}{n} T(r, f(z))+S(r, f) .
$$

Since $|q|>1$, by Lemma 2.3 we have $\rho(f) \leq \frac{\log (s j+s+1)-\log n}{\log |q|}$.

Since $\varphi_{1}(z)$ is a rational function, we can choose a sufficiently large constant $R(>0)$ such that $\varphi_{1}(z)$ has no zeros or poles in $\{z \in \mathbb{C}:|z|>R\}$. Since $f$ has infinitely many poles, we can choose a pole $z_{0}$ of $f$ of multiplicity $\tau \geq 1$ satisfying $\left|z_{0}\right|>R$. Then the right side of (3) has a pole of multiplicity $\tau_{1}=(s+1) \tau+s j$ at $z_{0}$. Then $f$ has a pole of multiplicity $\tau_{1}$ at $q z_{0}$. Replacing $z$ by $q z_{0}$ in (3), we see that $f$ has a pole of multiplicity $\tau_{2}=(s+1) \tau_{1}+s j$ at $q^{2} z_{0}$. We proceed to follow the steps above. Since $\varphi_{1}(z)$ has no zeros or poles in $\{z \in \mathbb{C}:|z|>R\}$ and $f$ has infinitely many poles again, we may construct poles $\zeta_{k}=q^{k} z_{0}, k \in \mathbb{N}_{+}$of $f$ of multiplicity $\tau_{k}$ satisfying

$$
\tau_{k}=(s+1) \tau_{k-1}+s j=(s+1)^{k} \tau+s j\left[(s+1)^{k-1}+\cdots+1\right],
$$

as $k \rightarrow \infty, k \in \mathbb{N}$. Since $|q|>1,\left|\zeta_{k}\right| \rightarrow \infty$ as $k \rightarrow \infty$. For sufficiently large $k$, we have

$$
\begin{aligned}
\tau(s+1)^{k} & \leq(\tau+j)(s+1)^{k}-j=\tau_{k} \leq \tau+\tau_{1}+\cdots+\tau_{k} \leq n\left(\left|\zeta_{k}\right|, f\right) \\
& \leq n\left(|q|^{k}\left|z_{0}\right|, f\right) .
\end{aligned}
$$

Thus, for each sufficiently large $r$, there exists a $k \in \mathbb{N}_{+}$such that

$$
r \in\left[|q|^{k}\left|z_{0}\right|,|q|^{(k+1)}\left|z_{0}\right|\right), \quad \text { i.e. } k>\frac{\log r-\log r_{0}-\log |q|}{\log |q|} .
$$


Thus, we have

$$
n(r, f) \geq \tau(s+1)^{k} \geq \tau(s+1)^{\frac{\log r-\log r_{0}-\log |q|}{\log |q|}} \geq K_{1}(s+1)^{\frac{\log r}{\log |q|},}
$$

where

$$
K_{1}=\tau(s+1)^{\frac{-\log r_{0}-\log |q|}{\log |q|}} .
$$

Since, for all $r \geq r_{0}$,

$$
K_{1}(s+1)^{\frac{\log r}{\log |q|}} \leq n(r, f) \leq \frac{1}{\log 2} N(2 r, f) \leq \frac{1}{\log 2} T(2 r, f),
$$

it follows from (11) that

$$
\rho(f) \geq \mu(f) \geq \frac{\log (s+1)}{\log |q|} .
$$

Thus, this completes the proof of Theorem 1.7.

\subsection{The proof of Theorem 1.8}

Since $\varphi_{2}(z)$ is a small function, similar to (7), we have

$$
T(|q| r, f(z)) \leq \frac{1}{n} T(r, f)+\frac{s}{n} T\left(r, f^{(j)}(z)\right)+S(r, f) .
$$

Since $f$ is a transcendental meromorphic function and $\bar{N}(r, f)=S(r, f)$, by Lemma 2.2 we have

$$
T\left(r, f^{(j)}(z)\right) \leq T(r, f)+S(r, f) .
$$

Thus, from (12) and (13), by using the same argument as in Theorem 1.6, we can get

$$
\rho(f) \leq \frac{\log (s+1)-\log n}{\log |q|} .
$$

If $n=1$ and $f$ has infinitely many poles, since the number of distinct common poles of $f$ and $\frac{1}{\varphi_{2}}$ is finite, we can choose a sufficiently large constant $R(>0)$ such that $f$ and $\frac{1}{\varphi_{2}(z)}$ have no common poles in $\{z \in \mathbb{C}:|z|>R\}$. Thus, we can take a pole $z_{0}$ of $f$ of multiplicity $\tau \geq 1$ satisfying $\left|z_{0}\right|>R$. By using the same argument as in Theorem 1.7, we can see that

$$
\rho(f) \geq \mu(f) \geq \frac{\log (s+1)}{\log |q|} .
$$

Hence, from (14) and (15), we complete the proof of Theorem 1.8.

\section{The proof of Theorem 1.9}

Since $f$ is a transcendental meromorphic solution of (5), and $\varphi_{3}(z)$ is a small function with respect to $f$, similar to the proof of (12), and by Lemma 2.2, we have

$$
T(r, f(p(z))) \leq \frac{s+s j+1}{n} T(r, f(z))+S(r, f)=\left(\frac{s+s j+1}{n}+o(1)\right) T(r, f) .
$$


Then, by Lemma 2.5, for any $\beta>1$ and for all $r>r_{0}$, we have

$$
T(r, f(p(z))) \leq\left(\frac{s+s j+1}{n}+o(1)\right) T(\beta r, f)
$$

Since $p(z)$ is a polynomial with $\operatorname{deg}_{z} p(z)=k \geq 2$, by Lemma 2.4 , for given $0<\delta<\left|p_{k}\right|$, let $\mu=\left|p_{k}\right|-\delta$, for given $\varepsilon>0$ and for sufficiently large $r$, it follows for (16) that

$$
(1-\varepsilon) T\left(\mu r^{k}, f\right) \leq\left(\frac{s+s j+1}{n}+o(1)\right) T(\beta r, f)
$$

Set $R=\beta r$, then we have

$$
(1-\varepsilon) T\left(\mu \beta^{-k} R^{k}, f\right) \leq\left(\frac{s+s j+1}{n}+o(1)\right) T(R, f) .
$$

Since $n<s+s j+1$ and $\beta>1, \mu>0$, we have $\frac{s+s j+1}{n}>1$ and $\mu \beta^{-k}>0$. Thus, by Lemma 2.6, letting $\varepsilon \rightarrow 0$ and $\beta \rightarrow 1$, we have

$$
T(r, f)=O\left((\log r)^{\alpha}\right), \quad \alpha=\frac{\log (s j+s+1)-\log n}{\log k} .
$$

Thus, this completes the proof of Theorem 1.9.

\section{The proof of Theorem 1.11}

Suppose that $f$ is a transcendental meromorphic solution of (6). Since $f$ has infinitely many poles, we can take a pole $z_{0}$ of $f$ of multiplicity $\tau \geq 1$. Since $d \geq 4$, we see that the right side of (6) has a pole of multiplicity $d \tau$ at $z_{0}$. Then it follows that $q z_{0}$ is a pole of $f$ of multiplicity $\tau_{1}=d \tau-\tau-1$. Since $d \geq 4$ and $\tau \geq 1$, we have $\tau_{1} \geq 1$. Replacing $z$ by $q z_{0}$ in (6), we have

$$
f\left(q^{2} z_{0}\right) f^{\prime}\left(q z_{0}\right)=R\left(q z_{0}, f\left(q z_{0}\right)\right) .
$$

Thus the right side of (18) has a pole of multiplicity $d \tau_{1}$ at $q z_{0}$. Then we see that $q^{2} z_{0}$ is a pole of $f$ of multiplicity $\tau_{2}=d \tau_{1}-\tau_{1}-1=(d-1)^{2} \tau-(d-1)-1$.

We proceed to follow the steps above. Since $f$ has infinitely many poles, we may construct poles $\zeta_{k}=q^{k} z_{0}, k \in N_{+}$of $f$ of multiplicity $\tau_{k}$ satisfying

$$
\begin{aligned}
\tau_{k} & =d \tau_{k-1}-\tau_{k-1}-1=(d-1)^{k} \tau-(d-1)^{k-1}-\cdots-(d-1)-1 \\
& =(d-1)^{k} \tau-\frac{(d-1)^{k}-1}{d-2}>(d-1)^{k}\left(\tau-\frac{1}{d-2}\right) .
\end{aligned}
$$

Since $\tau \geq 1$ and $d \geq 4, \tau-\frac{1}{d-2}>0$. Thus, since $\left|\zeta_{k}\right| \rightarrow \infty$ as $k \rightarrow \infty$, for sufficiently large $k$, we have

$$
(d-1)^{k}\left(\tau-\frac{1}{d-2}\right)<\tau_{k} \leq \tau_{1}+\tau_{2}+\cdots+\tau_{k} \leq n\left(\left|\zeta_{k}\right|, f\right) \leq n\left(|q|^{k}\left|z_{0}\right|, f\right) .
$$


Thus, for each sufficiently large $r$, there exists a $k \in \mathbb{N}_{+}$such that $r \in\left[|q|^{k}\left|z_{0}\right|,|q|^{k+1}\left|z_{0}\right|\right)$. By using the same method as in the proof of Theorem 1.7, from (20), we have

$$
\begin{aligned}
n(r, f) & \geq(d-1)^{k}\left(\tau-\frac{1}{d-2}\right) \geq(d-1)^{\frac{\log r-\log \left|z_{0}\right|-\log |q|}{\log |q|}}\left(\tau-\frac{1}{d-2}\right) \\
& \geq K_{2}(d-1)^{\frac{\log r}{\log |q|}}
\end{aligned}
$$

where

$$
K_{2}=\left(\tau-\frac{1}{d-2}\right)(d-1)^{\frac{-\log \left|z_{0}\right|-\log |q|}{\log |q|}} .
$$

Since for all $r \geq r_{0}$, we have

$$
K_{2}(d-1)^{\frac{\log r}{\log |q|}} \leq n(r, f) \leq \frac{1}{\log 2} N(2 r, f) \leq \frac{1}{\log 2} T(2 r, f) .
$$

Thus, it follows that

$$
\rho(f) \geq \mu(f) \geq \frac{\log (d-1)}{\log |q|}
$$

Thus, this completes the proof of Theorem 1.11 .

\section{Competing interests}

The authors declare that they have no competing interests.

\section{Authors' contributions}

HYX completed the main part of this article, HYX, LZY, and HW corrected the main theorems. All authors read and approved the final manuscript.

\section{Author details}

'Department of Informatics and Engineering, Jingdezhen Ceramic Institute, Jingdezhen, Jiangxi 333403, China.

2 Department of Mathematics, Shandong University, Jinan, Shandong 250100, P.R. China.

\section{Acknowledgements}

The authors thank the referee for his/her valuable suggestions to improve the present article.

The first author was supported by the NSF of China $(11301233,61202313)$, the Natural Science Foundation of Jiangxi Province in China (20132BAB211001, 20151BAB201008), and the Foundation of Education Department of Jiangxi (GJJ14644) of China. The second author was supported by the NSF of China (11371225, 11171013, and 11041005).

Received: 29 December 2014 Accepted: 7 April 2015 Published online: 10 June 2015

\section{References}

1. Hayman, WK: Meromorphic Functions. Clarendon, Oxford (1964)

2. Yang, L: Value Distribution Theory. Springer, Berlin (1993)

3. Yi, HX, Yang, CC: Uniqueness Theory of Meromorphic Functions. Kluwer Academic, Dordrecht (2003). Chinese original, Science Press, Beijing (1995)

4. Ritt, J: Transcendental transcendency of certain functions of Poincaré. Math. Ann. 95, 671-682 (1925/26)

5. Rubel, L: Some research problems about algebraic differential equations. Trans. Am. Math. Soc. 280(1), 43-52 (1983)

6. Ishizaki, K: Hypertranscendency of meromorphic solutions of a linear functional equation. Aequ. Math. 56(3), 271-283 (1998)

7. Wittich, H: Bemerkung zu einer Funktionalgeichungen von H. Poincaré. Arch. Math. (Basel) 2, 90-95 (1949/50)

8. Gundersen, GG, Heittokangas, J, Laine, I, Rieppo, J, Yang, D: Meromorphic solutions of generalized Schröder equations. Aequ. Math. 63, 110-135 (2002)

9. Beardon, AF: Entire solutions of $f(k z)=k f(z) f^{\prime}(z)$. Comput. Methods Funct. Theory 12(1), 273-278 (2012)

10. Zhang, GW: On a question of Beardon. J. Inequal. Appl. 2013, Art. 331 (2013)

11. $\mathrm{Ai}, \mathrm{L}, \mathrm{Yi}, \mathrm{CF}$ : The growth for solutions of a class of higher order linear differential equations with meromorphic coefficients. J. Jiangxi Norm. Univ., Nat. Sci. 38(3), 250-253 (2014)

12. Bergweiler, W, Ishizaki, K, Yanagihara, N: Growth of meromorphic solutions of some functional equations I. Aequ. Math. 63(1/2), 140-151 (2002) 
13. Halburd, R, Korhonen, R: Finite-order meromorphic solutions and the discrete Painlevé equations. Proc. Lond. Math. Soc. 94, 443-474 (2007)

14. Halburd, R, Korhonen, R: Nevanlinna theory for the difference operator. Ann. Acad. Sci. Fenn., Math. 31, 463-478 (2006)

15. Laine, I, Rieppo, J, Silvennoinen, H: Remarks on complex difference equations. Comput. Methods Funct. Theory 1 , 77-88 (2005)

16. Silvennoinen, H: Meromorphic solutions of some composite functional equations. Ann. Acad. Sci. Fenn., Math. Diss. $13,14-20(2003)$

17. Tu, J, Huang, $\mathrm{HX}, \mathrm{Xu}, \mathrm{HY}$, Chen, CF: The order and type of meromorphic functions and analytic functions in the unit disc. J. Jiangxi Norm. Univ., Nat. Sci. 37(5), 449-452 (2013)

18. Wang, J: Growth and poles of meromorphic solutions of some difference equations. J. Math. Anal. Appl. 379, 367-377 (2011)

19. Zhang, JL, Korhonen, R: On the Nevanlinna characteristic of $f(q z)$ and its applications. J. Math. Anal. Appl. 369 537-544 (2010)

20. He, J, Zheng, XM: The iterated order of meromorphic solutions of some classes of higher order linear differential equations. J. Jiangxi Norm. Univ., Nat. Sci. 36(6), 584-588 (2012)

21. Barnett, DC, Halburd, RG, Korhonen, RJ, Morgan, W: Nevanlinna theory for the $q$-difference operator and meromorphic solutions of q-difference equations. Proc. R. Soc. Edinb., Sect. A, Math. 137, 457-474 (2007)

22. Zheng, XM, Chen, ZX: Some properties of meromorphic solutions of $q$-difference equations. J. Math. Anal. Appl. 361, 472-480 (2010)

23. Heittokangas, J, Korhonen, R, Laine, I, Rieppo, J, Tohge, K: Complex difference equations of Malmquist type. Comput. Methods Funct. Theory 1(1), 27-39 (2001)

24. Laine, I: Nevanlinna Theory and Complex Differential Equations. de Gruyter, Berlin (1993)

25. Goldstein, R: Some results on factorization of meromorphic functions. J. Lond. Math. Soc. 4(2), 357-364 (1971)

26. Bank, S: A general theorem concerning the growth of solutions of first-order algebraic differential equations. Compos. Math. 25, 61-70 (1972)

27. Gundersen, GG: Finite order solutions of second order linear differential equations. Trans. Am. Math. Soc. 305, 415-429 (1988)

28. Chen, ZX, Huang, ZB, Zheng, XM: On properties of difference polynomials. Acta Math. Sci. 31B(2), 627-633 (2011)

29. Goldstein, R: On meromorphic solutions of certain functional equations. Aequ. Math. 18, 112-157 (1978) 